\title{
Some Considerations on the Energy Deposition During a RIA Transient Based On Monte Carlo Simulations
}

\author{
Julia Bartos ${ }^{1}$, Adrien Gruel ${ }^{1}$, Claire Vaglio-Gaudard ${ }^{2}$ and Christine Coquelet_Pascal ${ }^{1}$ \\ ${ }^{1}$ CEA, DES,IRESNE,DER,SPESI,LP2E, Cadarache, 13108 Saint Paul lez Durance, France \\ ${ }^{2}$ CEA, DES,IRESNE,DER,SESI, Cadarache, 13108 Saint Paul lez Durance, France \\ Corresponding author: julia.bartos@cea.fr
}

\begin{abstract}
Specific research reactors are capable of reproducing reactivity injection accidents in order to study the behavior of the nuclear fuel pins in accidental situations. In the CABRI research reactor, the fuel pin to be examined (test pin) is placed in the center of the core in a dedicated test loop. It is then subjected to a power transient, obtained by the fast depressurization of the ${ }^{3} \mathrm{He}$ neutron absorber gas from the transient rods located in the core. One of the central parameters of the experiment is the energy deposition in the test pin, which is currently not measured during a transient. Instead, it is assumed that the relative energy distribution between the core and the test pin is constant regardless the operational state of the reactor. Currently, this correlation is measured in steady state. As such, the impact of the variations in the neutron flux, fuel and moderator temperatures during the transient is assumed equivalent on the energy deposition in the core and in the test pin. The goal of this work is to improve our knowledge on the mechanisms involved in the transient energy deposition. The aim of this paper is to present a methodological approach for the energy deposition estimation during a CABRI transient, based on static Monte Carlo calculations. The results suggest that the transient energy deposition rate is mainly dependent on the helium pressure and the Doppler feedback, and the relative energy distribution between the core and test pin changes during the transient.
\end{abstract}

Keywords - Energy deposition, CABRI, RIA, Monte Carlo calculations, TRIPOLI4

\section{INTRODUCTION}

The term Reactivity Initiated Accident (RIA) refers to nuclear accidents involving a sudden and unexpected reactivity increase in the core. It is characterized by an extremely rapid power excursion with a significant energy deposition in the fuel assemblies surrounding the perturbation. In order to minimize the possibility of fuel failure and its consequences (such as the fuel dispersal in the primary circuit), the fuel safety criterion associated with this kind of accident is defined as a limit on the energy deposited in fuel pins [1].

Specific research reactors are capable of reproducing reactivity injection accidents in order to study the behavior of the nuclear fuel pins in accidental situations. In the CABRI research reactor [2], funded by the French Institute for Radioprotection and Nuclear Safety (IRSN) and located in the Cadarache research center of the French Alternative Energies and Atomic Energy Commission (CEA), the fuel pin to be examined (test pin) is placed at the center of the core, in a dedicated test loop, and is then subjected to a power transient obtained by the fast depressurization of the neutron absorber ${ }^{3} \mathrm{He}$ gas from the transient rods located in the core. The main parameters of the power pulse such as the peak power, the pulse length and the total energy deposition, depend on the operational characteristics of the reactor and are adjustable by experimental design.

The energy deposited in the test fuel pin during the transient is currently not measured. It is usually derived from the core characteristics at steady state, assuming a constant relative power distribution in the core and the test pin during all operational states of the reactor. The aim of this work is to improve our knowledge of the mechanisms involved in the energy deposition and relative energy distribution during a transient with static Monte Carlo calculations.

The transient is simulated by running several static calculations with different core configurations representative of the different stages of the transient. In order to define these core configurations, the different parameters (physical, experimental or computational) that could influence the relative energy distribution between the core and the test pin are first identified. This step is followed by a sensitivity study to determine the main parameters that the energy deposition depends on.

The methodologies currently used for estimating the energy deposition along with the summary of the parameters of interest is presented in section II. The results of the sensitivity studies are discussed in section III.

\section{CAlculation Methodology}

The methodology presented in this section is based on the use of the TRIPOLI-4® 3D continuous energy Monte Carlo neutronics code developed by CEA for particle transport calculations [3]. The required nuclear data, such as cross sections and fission spectra come from the JEFF-3.1.1. evaluation file [4]. 
The reactor core is accurately modeled with a $3 \mathrm{D}$ native TRIPOLI-4 geometry (see Fig. 1), and the initial core configuration corresponds to the critical state of the CABRI reactor at $100 \mathrm{~kW}$ with a helium pressure of 11.4 bar in the transient rods. The test pin is a fresh $6 \%$ enriched $\mathrm{UO}_{2}$ pin, identical to those found in the core. The initial moderator parameters in the pressurized water loop are considered identical to the core: water at ambient pressure and $20^{\circ} \mathrm{C}$.
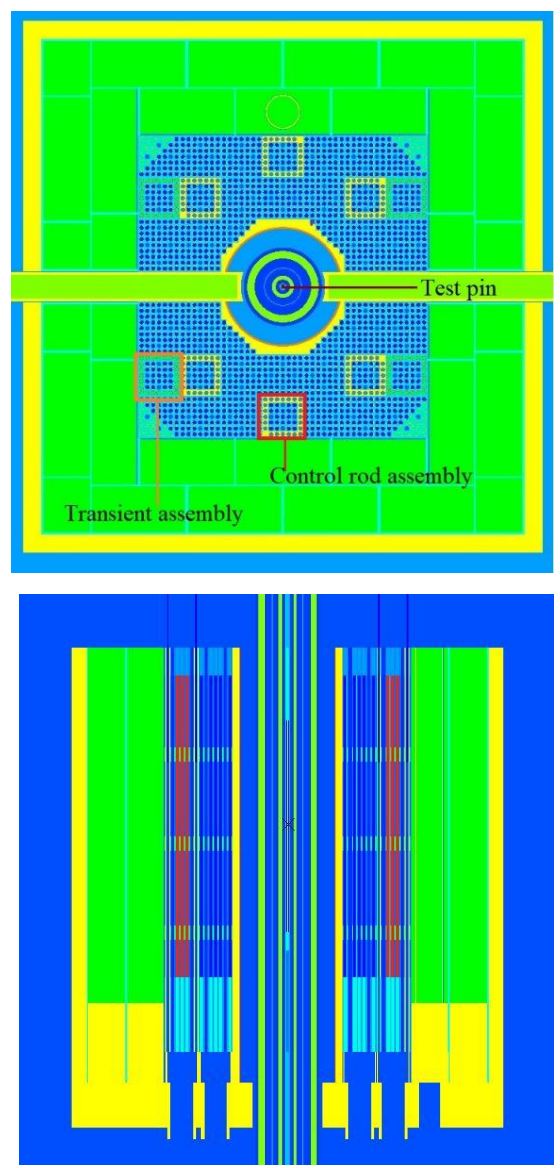

Fig. 1. Radial (top) and axial (bottom) view of the CABRI reactor core

\section{A. Estimation of the Energy Deposition in Steady-State}

There are two approaches for calculating the energy deposition in steady-state with TRIPOLI-4.

In the first approach, the energy deposition is estimated based on the fission energy generated in the core and in the test pin. First, the fission rates $\left(\tau_{\text {fission }, i}\right)$ are computed with TRIPOLI$4 ®$ for each fissile isotope $i$ both in the core and in the test pin. The power generated is then calculated by taking into account the individual contribution of each fissile isotope based on their reaction rate and their fission energy $\left(E_{\text {fission. } i}\right)^{1}$, as shown in (1). Finally, the energy deposition can be calculated by integrating the core and the pin power over the defined duration.

$$
P=\sum_{i} \tau_{\text {fission }, i} \times E_{\text {fission. } i}
$$

In this approach, however, it is assumed that the energy generated by fission, which appears as the kinetic energy of fission products, neutrons, $\gamma$ and $\beta$ particles is deposited entirely where the fission took place. Although the largest part of the generated energy is transmitted to the fission products, which convey their energy to the surrounding medium, that is to say the fuel pin, without being transported, the energy carried away by the prompt and delayed neutrons, $\gamma$ and $\beta$ particles can be deposited in the surrounding fuel pins, moderator or structural materials.

This spatial repartition of the energy generated during fission can be taken into account in the nuclear heating scheme. Nuclear heating is expressed in terms of energy deposition in a given volume.

The total nuclear heating is composed of: - prompt heating which comes from the kinetic energy of fission fragments, kinetic energy of neutrons, energy of prompt photons emitted by neutron interactions (mainly fission, radiative capture and inelastic scattering);

- delayed heating which comes from the energy deposition of delayed particles (mainly photons) emitted by the decay of fission and activation products.

The prompt and delayed heating can be estimated separately. The transport of 4 (neutrons, photons, electrons and positrons) or 3 particles (photons, electrons and positrons) is simulated in 3D TRIPOLI-4® calculations, depending whether the prompt or delayed heating is calculated. The electromagnetic cascade shower is modelled with both electrons and positrons in the TRIPOLI-4® calculations. The photon transport is made on the basis of the EPDL97 photo-atomic library developed at Lawrence Livermore National Laboratory [4].

The prompt heating is obtained by running a criticality calculation in TRIPOLI-4®. In this approach, the energy allocated to the charged particles and recoil nucleus is calculated by subtracting the energy carried away by transported particles from the initial energy released from fission. The energy deposition can be directly computed in TRIPOLI-4®.

Prior to calculating the delayed nuclear heating, the delayed source has to be defined. Given that TRIPOLI-4® does not model the decay of fission and activation products, the delayed $\gamma$ and $\beta$ sources associated with the decay of fission and activation products are determined with the DARWIN [5] depletion code. This source is then propagated with a fixedsource TRIPOLI-4® calculation, in which the delayed contribution to the nuclear heating is computed.

Table I presents the instantaneous energy deposition in the test pin (fuel and cladding) and in the core, normalized to $1 \mathrm{MW}$ core power and calculated with the two methodologies presented in this section. When estimated with the fission energy scheme, this value represents the energy generated by all fissions in the test pin and assumed to be deposited 
entirely within the pin. While when estimated with nuclear heating it represents the energy deposited in the test pin by taking into account the contribution to the energy deposition of the particles coming from the core and the energy 'loss' due to the particles that migrate outside the pin, and thus deposit their energy elsewhere.

TABLE I

ENERGY DEPOSITION VALUES IN THE TEST PIN, NORMALIZED TO 1MW CORE POWER, CALCULATED WITH THE FISSION ENERGY AND THE NUCLEAR HEATING SCHEME

\begin{tabular}{|c|c|c|c|}
\hline \multicolumn{2}{|c|}{ Fission energy scheme } & \multicolumn{2}{c|}{ Nuclear heating scheme } \\
\hline $\begin{array}{c}\text { Power deposition } \\
\text { (W/MW) }\end{array}$ & $\begin{array}{c}\text { Uncertainty }{ }^{2} \\
(\%)\end{array}$ & $\begin{array}{c}\text { Power deposition } \\
\text { (W/MW) }\end{array}$ & $\begin{array}{c}\text { Uncertainty } \\
(\%)\end{array}$ \\
\hline 2113 & 0,1 & 1948 & 0,3 \\
\hline
\end{tabular}

Table II presents the decomposition of the energy deposition. The majority of the energy deposition is due to the fissions taking place in the pin ('pin to pin'), however, as suggested from prior calculations, $13 \%$ of the heating generated in the test pin is deposited outside of the test pin ('pin to outside'), while $1 \%$ of the deposited energy is due to the particles coming from the core. The delayed heating contribution in the test pin is around $3 \%$ of the total heating. In a power reactor in equilibrium cycle condition, this value is about $7 \%$. The discrepancy is due to the short irradiation duration considered for these calculations (5 minutes).

TABLE II

DECOMPOSITION OF ENERGY DEPOSITION BY SPATIAL DISTRIBUTION AND BY PROMPT AND DELAYED COMPONENTS, NORMALIZED TO 1MW CORE POWER

\begin{tabular}{|c|c|c|c|}
\hline \multirow{2}{*}{ Pin to pin } & $\begin{array}{c}\text { Prompt energy } \\
\text { deposition } \\
\text { (W/MW) }\end{array}$ & \multicolumn{2}{|c|}{$\begin{array}{c}\text { Delayed energy deposition } \\
\text { (W/MW) }\end{array}$} \\
\cline { 3 - 4 } & $1878 \pm 0,3 \%$ & $\begin{array}{c}\text { Gamma } \\
\text { (W/MW) }\end{array}$ & $\begin{array}{c}\text { Beta } \\
\text { (W/MW) }\end{array}$ \\
\hline Core to pin & $15 \pm 0,75 \pm 0,03 \%$ & $43,75 \pm 0,02 \%$ \\
\hline Pin to outside & $250 \pm 1,4 \%$ & $1,57 \pm 3 \%$ & $0,13 \pm 4 \%$ \\
\hline & & $28,22 \pm 0,03 \%$ & $4,69 \pm 0,02 \%$ \\
\hline
\end{tabular}

Even though there are different approaches for estimating the energy deposition, all methodologies are derived from the same physical quantities, calculated in each case with TRIPOLI-4®, the neutron flux and reaction rates. Since the stationary transport equation is solved, the impact of the variation of the neutron flux and spectra during the transient is not taken into account.

Further approximations are introduced by the fact that the kinetic parameters and Doppler coefficient are considered constant, whereas these parameters may vary during the transient. Moreover, neither calculation scheme takes into account the thermal-hydraulic feedbacks and the thermomechanical behavior of the fuel during the transient.

\footnotetext{
${ }^{2}$ The uncertainty values presented in this paper refer to the Monte Carlo statistical uncertainty, at one standard deviation
}

The possible impacting parameters on the energy deposition are summarized in the following section.

\section{B. Identification of the Impacting Factors on the Transient Energy Deposition}

The impacting parameters on the transient energy deposition can be classified as experimental, physical or calculation parameters.

In the case of the CABRI reactor, the main parameters characterizing the power pulse such as the peak power, the pulse length and the total energy deposition, depend on the operational characteristics of the reactor and are adjustable by experiment design. The initial pressure of the ${ }^{3} \mathrm{He}$ gas and the kinetics of the depressurization determine the maximum core power attained during the test and the duration of the transient while the total energy is finely tuned with the timing of the control rod drop. Similarly, the choice in the test pin, i.e. UOX or MOX pin, its burnup or enrichment can also influence the energy deposition during a transient.

The helium depressurization leads to a rapid power excursion (length of the power pulse between 8 to $100 \mathrm{~ms}$ ) and instantaneous temperature increase in the core. The resulting fuel temperature increase (in the order of hundreds of $\mathrm{K}$ ) terminates the power surge due to the Doppler feedback. The impact of the increase of the moderator temperature and the subsequent density decrease in both the core and the test loop, although slower and less important in amplitude, needs also to be considered.

The reactor core, including the test loop, is accurately modelled with a 3D native TRIPOLI-4® geometry. However, there are several approximations made in the modelling of the fuel pins. The isotopic composition of the fuel, as well as the radial temperature profile within the fuel pins, is considered homogeneous. Yet, for instance in the case of a test fuel with a high burnup, the initial uniform fissile material distribution is changed due to the enhanced neutron resonance capture on the pellet periphery, leading to a local buildup of transuranium isotopes at the pellet outer ring, called the rim region. The radial temperature profile also varies during a transient as discussed in [6]. In addition, local geometry changes in the pellet and/or clad during the transient may affect the heat exchange between the different media, that could lead to rapidly varying temperatures.

In conclusion, the main impacting parameters on the energy deposition and its calculation are the following:

- Experimental parameters:

- initial helium pressure and depressurization kinetics (short or long pulse)

- $\quad$ test pin characteristics (UOX or MOX fuel, burnup and enrichment)

- $\quad$ Physical parameters:

- reactivity, thermomechanical and thermalhydraulics feedbacks 
- Simulation parameters:

- modelling approximations (concerning the temperature and isotopic concentration discretization for the fuel pin), nuclear data library

- calculation methodology: estimation based on fission energy or based on nuclear heating

- computational scheme: static or kinetic Monte Carlo calculation

The sensitivity analysis presented in this paper is focused on the variations in both the physical and experimental parameters, more precisely the helium pressure and fuel and moderator temperature, and their impact on the energy deposition during a transient, presented in the next section.

\section{SENSITIVITY STUDIES ON THE TRANSIENT ENERGY DEPOSITION}

The dynamic aspect of the transient is approximated with static TRIPOLI-4® calculations by running several calculations with different core configurations, i.e. simulation points, representative of the different stages of the transient. At each of these simulation points the helium pressure, the fuel and moderator temperatures are modified. The aim of this approach is to account for the reactivity variation (due to both the helium pressure and the reactivity feedbacks) associated with the studied transient, in order to determine the energy deposition during the transient. Given that this is a sensitivity analysis, it was assumed that the simplified approach relying on static calculations is relevant for the identification of the parameters influencing the energy deposition. Then at each simulation point, an instantaneous energy deposition is estimated based on the fission energies, as shown in (1).

Fig. 2 presents the characteristics of the hypothetical transient used for this study. The transient characteristics, such as the helium depressurization, the average fuel temperature and core water temperature variations are obtained with the SPARTE code. SPARTE [2] (Simulation Prediction and Analysis for RIA Transients an Excursions) is a recently developed code dedicated to R\&D studies on the physical characteristics of the CABRI transients, such as the power peak, transient length and power trace.

The moderator temperature and consequently the moderator density variation is considered identical during the transient. SPARTE computes one average transient temperature profile for all the fuel pins in the core. Given that the test pin is identical to the core fuel pins, it is assumed that the temperature profile of the test pin can be reproduced based on the temperature profile of the pin with the highest radial form factor.

\section{A. Variations in Energy Generation Induced by the ${ }^{3} \mathrm{He}$ Depressurization}

As described in chapter II, the energy deposition is estimated based on the reaction rates and fission energies. In order to understand the behavior of the energy deposition in the test pin during the depressurization, we have to study the normalized fission rate distribution in the core.

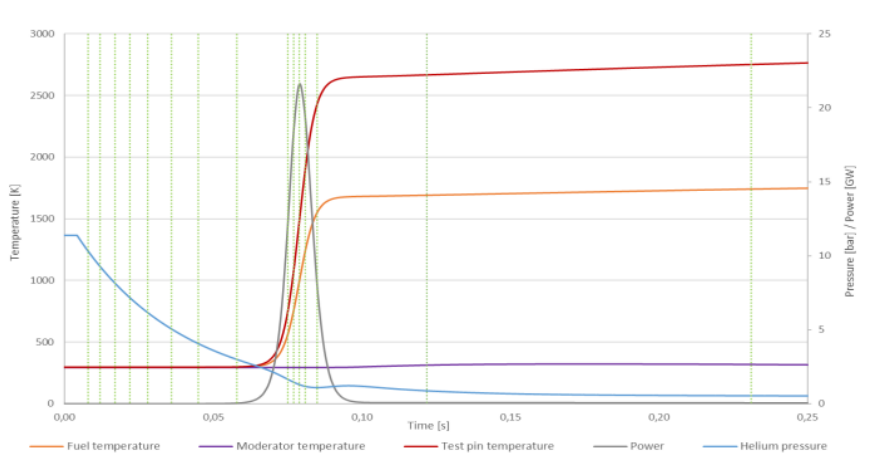

Fig. 2. Pressure (blue) and temperature (red for the test pin and orange for the pins in the core) during the studied transient and the chosen simulation points. Results obtained with the SPARTE code

At each simulation point, the reaction rates are normalized to the average fission rate. This normalization is applied on all fission distribution results presented in this paper.

Fig. 3 (left) shows that in steady state, prior to the depressurization, the reaction rate is peaked in the center of the core, with the lowest values in the transient rods and in the periphery due to the increased absorption and neutron leakage in those regions. On the right-hand side figure, this peak is observable even after the depressurization, however it is less important, given that the fission rate in the transient rods increases by $\sim 60 \%$, while there is no significant change in the fission rate in the rods close to the center. Concerning the test pin, the fission rate decreases by $4 \%$ between the two states.
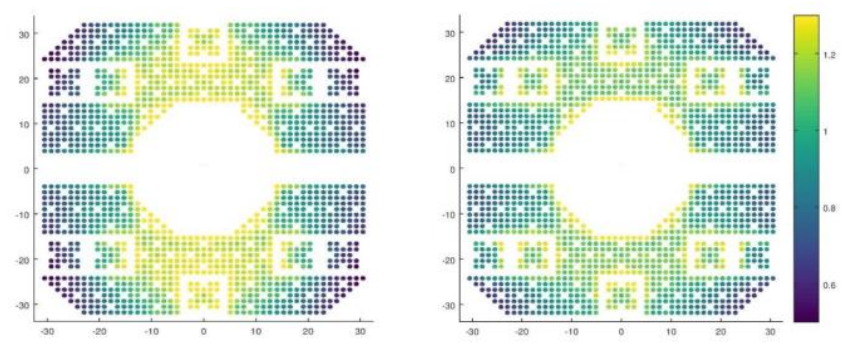

Fig. 3. Fission rate distribution in the core at steady state (left) and after the helium depressurization (right). Values normalized to the $\mathrm{k}_{\mathrm{eff}}$ and the average fission rate

Fig. 4 depicts the energy deposition in the core and test pin estimated from fission energies according to the helium pressure. As suggested by the fission rate variations, the energy deposition in the core is monotonically increasing during the helium depressurization, due to the increased fission rate in the transient rods. The deposition rate in the test pin however, is decreasing, as suggested by the flux variation. The statistical relative uncertainties are within the marker size, around $0.2 \%$. 


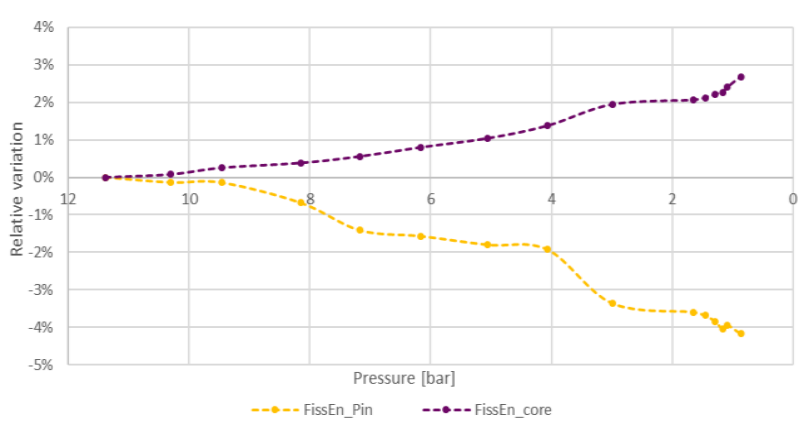

Fig. 4. Relative variation of the energy deposition in the core and test pin according to the helium pressure

\section{B. Variation in Energy Generation Induced by Doppler Feedback}

The helium depressurization leads to an extremely rapid power excursion and fuel temperature increase. With the increasing temperatures the neutron flux decreases as a result of the Doppler broadening of the resonance capture cross section. The individual impact of the Doppler feedback can be seen on Fig. 5. For these simulations, the helium pressure was kept constant while the temperature increased according to Fig. 3.

Contrary to the helium depressurization, the Doppler feedback has the same impact on the energy deposition rate in the core and in the test pin. While the depressurization has local effects in the reaction rates in the core (see Fig. 3), the Doppler feedback has a global and instantaneous impact.

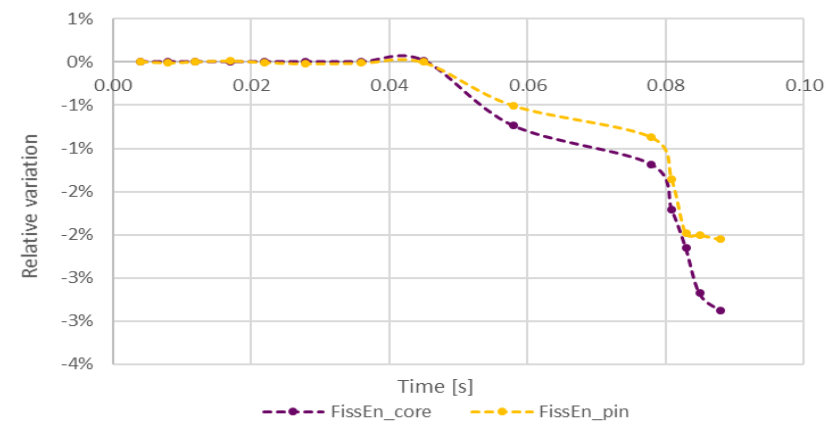

Fig. 5. Relative variation of the energy deposition in the core and test pin due to the Doppler feedback during the transient

As Fig. 6 shows, the fission rate distribution itself does not change with the increasing temperature. It is to be noted, that in the current calculation scheme, each fuel pin in the core is assumed to have the same temperature. This approximation needs to be improved in further studies.
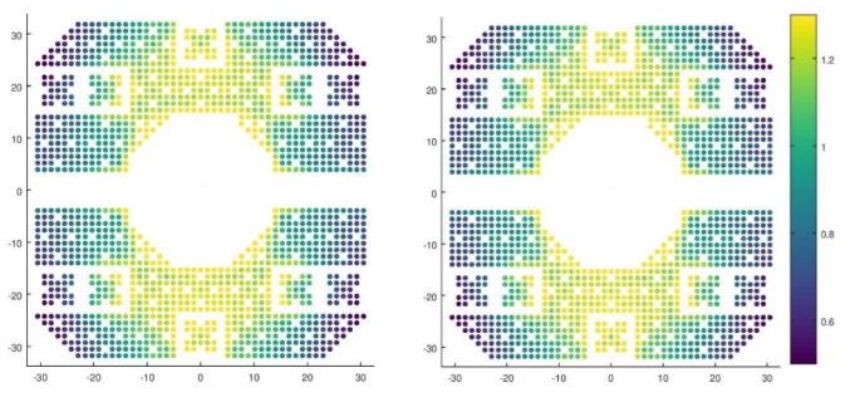

Fig. 6. Fission rate distribution at steady state (left) and after the transient (right), normalized to the $\mathrm{k}_{\text {eff }}$ and average fission rate

\section{Variations in Energy Generation Induced by Moderator Feedback}

The moderator temperature increase, and consequently its density decrease appears with a delay compared to the Doppler feedback. As discussed in [7] it takes around $150 \mathrm{~ms}$ for heat transfer to take place between the fuel and the surrounding moderator (Fig. 8). Given the duration of the studied transient, $9 \mathrm{~ms}$, the moderator effect is not relevant for the energy generation during the transient. As Fig. 7 shows, the relative variation in the energy deposition is under $0.3 \%$, which is within the convergence uncertainty of the calculations.

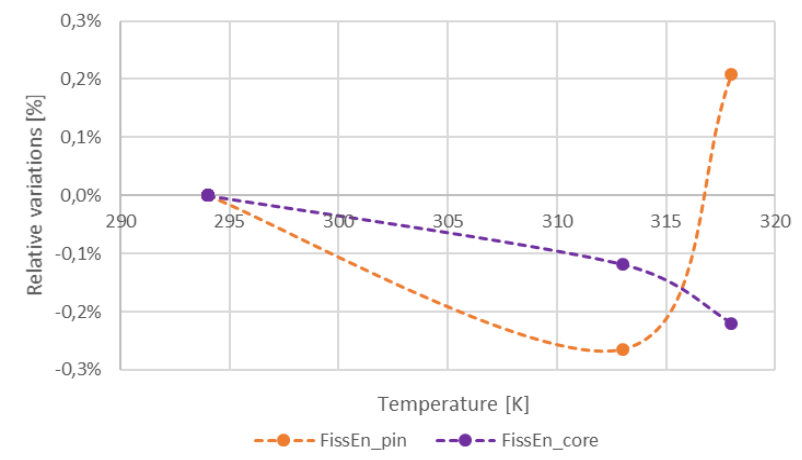

Fig. 7. Relative variation in the energy deposited in the core and in the test pin versus the moderator temperature increase

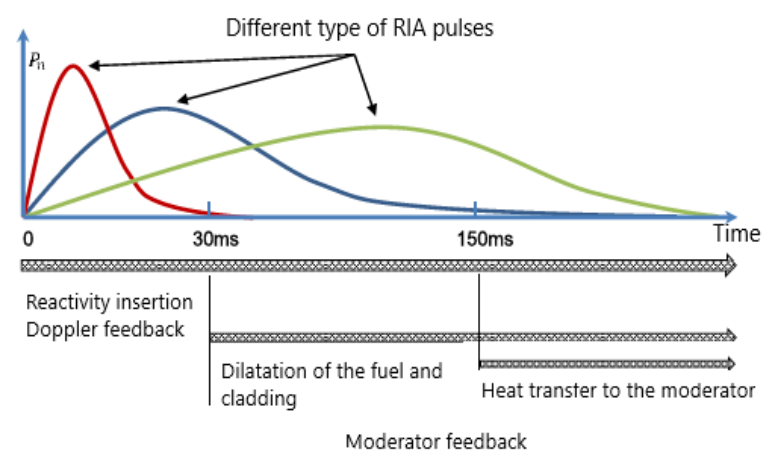

Fig. 8. Chronology of the reactivity feedbacks depending on the length of the transient [7] 


\section{Variations in Energy Generation During a Transient}

Fig. 9 presents the relative variation of the energy deposition in the test pin and in the core according to the time. Given that these are static calculations, the time and the simulation instants actually represent a set of values for the initial parameters (helium pressure, fuel and moderator temperature) representative of different stages of the hypothetical transient chosen to study.

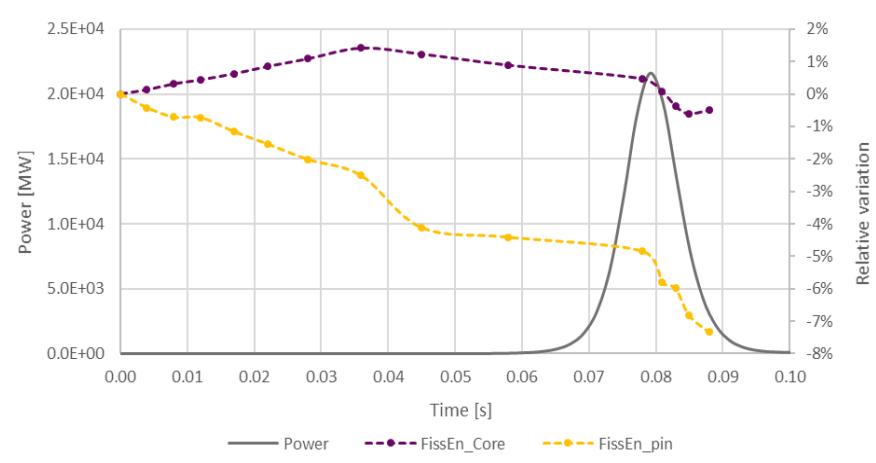

Fig. 9. Relative variation in the energy deposition in the core and test pin during the transient

The general trend in the test pin is the decrease of the energy deposition, all throughout the transient. Special attention needs to be given to the variations within the power peak itself, since the major part of the energy deposition happens during the power peak. When considering the power peak, current results suggest that the most impacting parameter on the energy deposition is the Doppler feedback. The energy deposition rate during the peak decreases by $0.5 \%$ when only the helium pressure varies, while it decreases by $2 \%$ when the fuel temperature increases. The corresponding relative uncertainties are around $0.2 \%$ (within the marker size).

Whereas, on the core level, the helium depressurization and the Doppler feedback have opposite effect on the energy deposition. During the early stages of the transient the energy deposition rate is increasing due to the lower helium pressure, however, as soon as the fuel starts to heat up, the Doppler feedback and the resulting increased absorption rate lead to a steady decrease in the energy deposition rate. This decrease is more important during the power peak, according to the jump in the temperature increase.

\section{CONCLUSIONS AND PERSPECTIVES}

This paper presents methodological elements, based on impact studies, aimed at improving our knowledge of the mechanisms involved in the energy deposition during a transient with static Monte Carlo calculations. The transient is modeled by running several static TRIPOLI-4® calculations with different core configurations representative of the different stages of the transient.

The steady state energy deposition is computed both based on fission energies and nuclear heating for the test case studied in order to study the limitation of the fission energy scheme concerning the spatial repartition of the deposited energy. In the first approach, the energy generated by the fissions in the test pin is assumed to be deposited locally. Computing the energy deposition with the nuclear heating scheme, which takes into account the spatial distribution of the energy, shows that the former approach overestimates the energy distribution in the pin by $11 \%$. When looking only at the energy distribution following a fission in the test pin, the calculations show that $13 \%$ of the energy generated is deposited outside the pin, while $1 \%$ is of the energy deposited in the test pin is due to the particles coming from the core. This is in accordance with the aforementioned overestimation.

Furthermore, in order to evaluate the most impacting physical parameters on the energy deposition during a transient, a sensitivity study is carried out. The results illustrate that the energy deposition is most dependent on the helium pressure variations and the Doppler feedback. A future objective of this study is to study the possible impact of other experimental parameters (control rod position, initial helium pressure, depressurization kinetics,..) as well as the impact of current modelling approximations on the energy deposition.

Although this approach does take into account the impact of the helium depressurization and of the resulting reactivity feedbacks, the dynamic behavior of the system can not be modelled with static calculations. The goal of the future calculations is to define the time-dependent energy deposition in the core and in the test pin during the transient with kinetic Monte Carlo simulations.

\section{REFERENCES}

[1] OECD, "Nuclear fuel behaviour under reactivity-initiated conditions, state-of-the-art report," NEA/CSNI/R(2010)1, 2010 .

[2] O. Clamens, P. Blaise, J.-P. Hudelot, J. Lecerf, B. Duc and B. Biard, "Coupled Experimental and Computational Approach for CABRI Power Transients Analysis," IEEE Transactions on Nuclear Science, vol. 65, no. 9, pp. 2434-42, 2018.

[3] E. Brun, F. Damian, C. Diop, F. Malvagi, O. Petit and A. Zoia, "TRIPOLI-4, CEA, EDF and AREVA reference Monte Carlo code," Annals of Nuclear Energy, vol. 82, pp. 151-160, 2015.

[4] A. Santamarina, B. Bernard and Y. Rugama, "The JEFF3.1.1 Nuclear Data Library, JEFF Report 22," 2009.

[5] A. Tsilanizara, C. M. Diop, B. Nimal and et al., "DARWIN: An EvolutionCode System for a Large Range of Applications," Journal of Nuclear Science and Technology, vol. 37, pp. 845-849, 2014.

[6] I. Guenot-Delahaie, J. Sercombe, T. Helfer, P. Goldbronn, E. Frederici , T. Le Jolu, A. Parrot, C. Delafoy and C. Bernaudat, "Simulation of RIA transients on UO2-M5 fuel rods with ALCYONE V1.4 fuel performance code," Water Reactor Fuel Performance Meeting Proceedings, 2017.

[7] J. Labit, N. Marie, J-P. Hudelot and E. Merle, "An advanced validation experimental methodology of multiphysics calculation tools on CABRI transients," in Proceedings of International Conference $M \& C$, Portland, USA, 2019. 